\title{
Functional Connectivity in Aging
}

Franziskus Liem¹, Linda Geerligs ${ }^{2}$, Jessica S. Damoiseaux ${ }^{3}$, Daniel S. Margulies ${ }^{4}$

Chapter to be published in

Schaie, K.W. \& Willis, S., (Eds.). (In Press). Handbook of the Psychology of Aging, 9e.

San Diego: Academic Press

\footnotetext{
${ }^{1}$ University Research Priority Program "Dynamics of Healthy Aging”, University of Zurich, Switzerland

${ }^{2}$ Donders Institute for Brain, Cognition and Behaviour, Radboud University, Nijmegen, The Netherlands

${ }^{3}$ Department of Psychology \& Institute of Gerontology, Wayne State University, Detroit, MI, USA

${ }^{4}$ CNRS UMR 7225, Frontlab, Institut du Cerveau et de la Moelle épinière (ICM), Paris, France
} 


\section{General introduction and outline of chapter}

A large body of research shows that aging is accompanied by localized changes in brain structure and function. However, over the past decade the neuroimaging community has begun to recognize the importance of investigating the brain as a network. Brain regions don't function independently, rather they form an expansive network that allows for communication between distant areas and enables complex cognitive functioning. Hence, age-related changes in the network structure might explain changes in cognitive functioning.

Characterizing this network by investigating the brain's functional connectivity has enabled new insights into brain organization. In this chapter, we will outline how the brain's functional connectivity is affected by aging and how changes in functional connectivity relate to changes in cognitive functioning. We will address how neurodegenerative pathology influences functional connectivity and how, based on these measurements, biomarkers for clinical outcome might be developed in the future.

\section{Functional connectivity}

Over the past decade, functional connectivity has emerged as a dominant MRI-based method for investigating human brain organization. The term was first introduced to the neuroimaging literature in an effort to clarify the various forms that connectivity could take. Unlike 'structural connectivity', referring to the anatomical paths between brain regions, and 'effective connectivity', referring to the causal influence of one region on the function of another, 'functional connectivity' refers a temporal relationship between the activity of two regions (Friston, 1994). For example, in the performance of a behavioral task such as finger tapping, regions whose activity covaries during tapping are described as functionally connected with one another. The observation that brain regions involved in the performance of a task are also correlated in their ongoing spontaneous activity, even during the baseline, 
resting state (Biswal, Yetkin, Haughton, \& Hyde, 1995), has given rise to the methodology that is at the heart of this chapter.

Resting-state functional magnetic resonance imaging (fMRI) refers to the study of ongoing, spontaneous brain activity that is always present, but more easily studied when research participants are simply asked to relax in the MRI scanner and not do anything in particular. The field emerged from two distinct lines of research in the late-1990s, leading to substantial confusion regarding terminology that merits clarification at the outset. One line of inquiry was the aforementioned study of spontaneous brain activity, and the observation that regions involved in the same functional systems are correlated in their intrinsic activity at rest, referred to as 'resting-state networks' (Beckmann, DeLuca, Devlin, \& Smith, 2005; Damoiseaux et al., 2006; De Luca, Beckmann, De Stefano, Matthews, \& Smith, 2006) (see Figure 1).

The other line of research addresses a set of brain regions that are more active during the baseline, 'resting', or 'default' state than they are during task performance (Raichle et al., 2001; Shulman et al., 1997). These regions subsequently became known as the 'default mode network' (Greicius, Krasnow, Reiss, \& Menon, 2003), which is one of several 'resting-state networks' (see Figure 1), and like all resting-state networks, it also exhibits characteristic activation patterns in response to task demands. In the case of the default mode network (DMN), one of its defining features is a consistent decrease in activity during goal-oriented externally demanding tasks. 


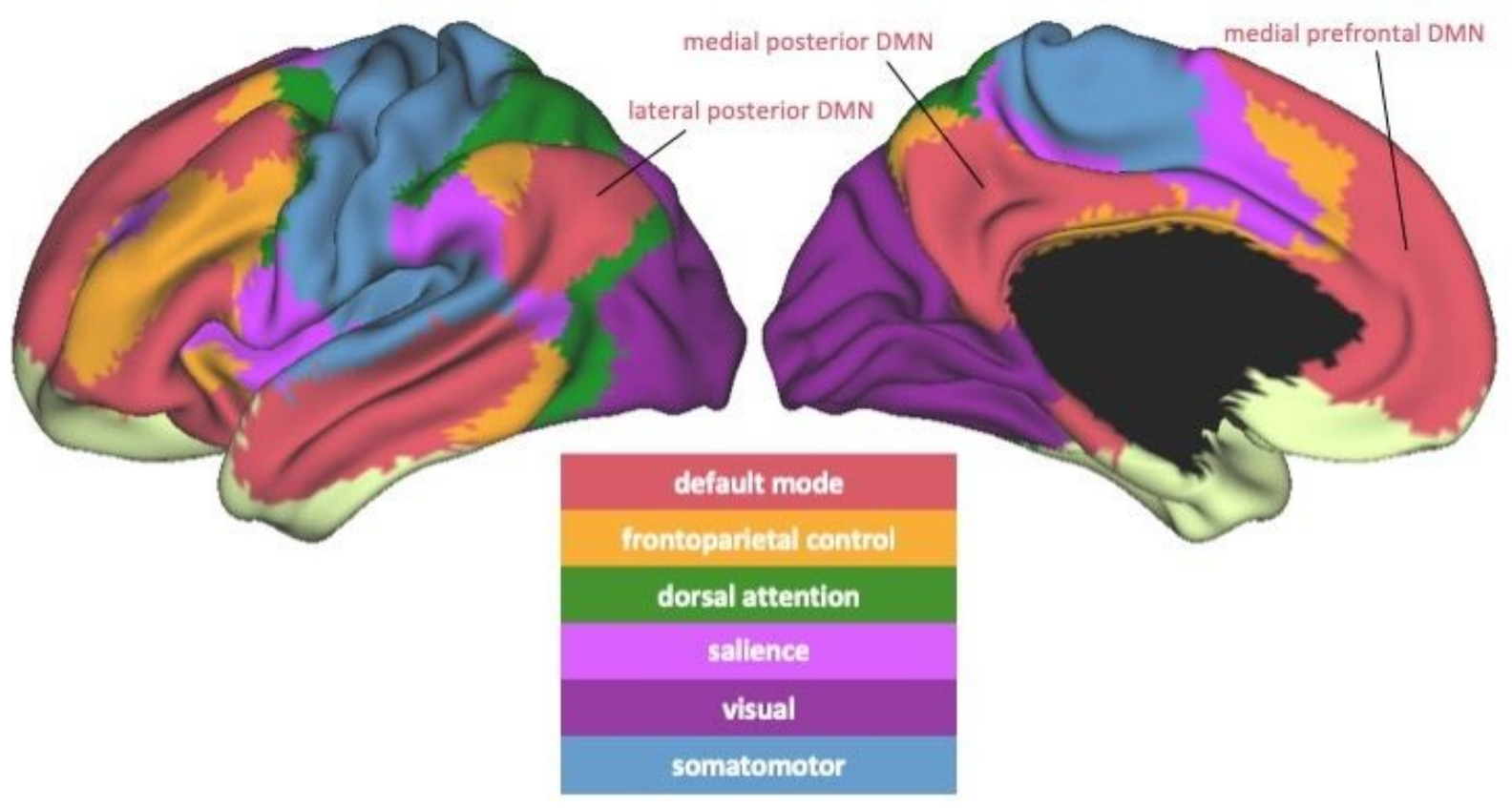

FIGURE 1: Aging-relevant canonical resting state networks according to Yeo et al. (2011) rendered on the surface of the left cortical hemisphere. Left: lateral view; right medial view. Prominent DMN-regions are highlighted.

\section{The default mode network}

The DMN is a network of great interest to both cognitive and clinical neuroscience because of its implication across a wide range of psychiatric disorders as well as its distinct functional properties. As mentioned before, early studies described this network as a set of regions that showed more activity when research participants viewed a blank screen or fixation cross as compared to performing a cognitively demanding task - known as 'task-related deactivation' (Raichle et al., 2001). In addition, regions of the default mode network also demonstrate significant functional connectivity with each other (Greicius et al., 2003). There are different definitions of regions that are included in the DMN, however the canonical regions include frontal (medial prefrontal cortex) and posterior (posterior cingulate cortex, precuneus) midline regions, and the lateral parietal cortex (see Figure 1). 
These initial findings led to the conclusion that the DMN represents a neutral, baseline, or "default mode" of brain function. Later studies further investigating its specific functional role linked DMN-regions to spontaneous cognition (daydreaming, mind wandering, self-referential processing, introspection) (Andrews-Hanna, Smallwood, \& Spreng, 2014), and learning and memory (Daselaar et al., 2009). Beyond these involvements, the DMN is assumed to have a fundamental role in brain function. This is demonstrated by the fact that the DMN can be found in non-human animals like rats (Lu et al., 2012) and monkeys (Vincent et al., 2007). Furthermore, DMN also is visible during states of consciousness without spontaneous cognition, for instance, anesthesia (Greicius et al., 2008). It is assumed that $\mathrm{DMN}$ regions serve as hubs that integrate information from the entire brain network (Greicius et al., 2008; Raichle, 2015).

\section{Functional connectivity in aging}

\section{Within-network connectivity}

Regions within the DMN become less functionally connected with age. This finding has been replicated across numerous studies using different methods. For instance, Andrews-Hanna et al. (2007) investigated age-related differences in the DMN by comparing a group of young adults and cognitively healthy older adults. Using a seed-based approach they quantified the connectivity between DMN seed regions and found that functional connectivity between most regions is significantly lower in older subjects. In an independent study, using a very different methodological approach, independent component analysis, Damoiseaux et al. (2008) compared DMN-connectivity for similar groups of subjects. They found two DMN components, one with a focus on posterior midline and lateral regions, one with a more anterior focus. The older group showed lower connectivity than the younger group in both components. The effect of lower DMN functional connectivity in older subjects 
has been replicated in other cross-sectional group studies as well (Geerligs, Renken, Saliasi, Maurits, \& Lorist, 2015; Grady, Sarraf, Saverino, \& Campbell, 2016). Furthermore, the robustness of these findings was corroborated by a recent meta-analysis that aggregated data from 30 independent studies (Mak et al., 2017).

Age-effects in DMN connectivity have been shown not only in cross-sectional but also in longitudinal datasets, demonstrating changes in the connectivity of individual brains over time - known as 'within-person' effects. Cross-sectional research designs aim to extrapolate from between-person effects to within-person effects, which is problematic (as discussed in more detail in the "Limitations" section). Therefore, longitudinal research designs can give unique insight into true within-subject change of functional connectivity. So far, only a few longitudinal studies investigate effects of aging within functional networks. For instance, $\mathrm{Ng}$ et al. (2016) found that DMN connectivity in older adults (approximately between 60 and 87 years old) decreases over four years (within-person effect), in addition to replicating cross-sectional age-related differences (between-person effect). Using a slightly wider age-range (49-87 years), a similar study showed that change in within-DMN connectivity follows an inverted u-shape depending on age. While participants below 66 years showed an increase in DMN connectivity over time, participants older than 74 years showed a decline. In contrast, other studies found stability over time (Persson, Pudas, Nilsson, \& Nyberg, 2014) or at least no significant age-related change (Fjell et al., 2015) in default mode network connectivity.

While the majority of research has assessed the overall strength of connectivity within the DMN as a whole, studies investigating the specific connectivity between pairs of DMN regions have found that connectivity between the anterior and posterior regions of the medial wall (e.g, medial prefrontal cortex, posterior cingulate cortex) are the most affected by aging (Andrews-Hanna et al., 2007; Mak et al., 2017). 
The other portion of the DMN that is particularly susceptible to aging is the medial temporal lobe (MTL) subsystem, which most prominently includes the hippocampus. The MTL has strong connections to medial 'core' DMN regions and is often considered part of the DMN. The MTL is vitally involved in memory function and is, therefore, a pivotal region for aging research. It is, for instance, very well established that hippocampal volume declines with age. That is true in cognitively unimpaired people (Fraser, Shaw, \& Cherbuin, 2015), as well as in patients with dementia (Pini et al., 2016). Several additional studies, described below, investigate the connectivity within MTL regions and between MTL and other DMN regions.

Functional connectivity between MTL and other cortical DMN regions decreases with age. This effect has been shown by cross-sectional (Damoiseaux, Viviano, Yuan, \& Raz, 2016; Salami, Pudas, \& Nyberg, 2014), as well as longitudinal studies (Salami, Wåhlin, Kaboodvand, Lundquist, \& Nyberg, 2016), but likely is specific to posterior parts of the MTL (Damoiseaux et al., 2016; Salami et al., 2016).

Such differences across the anterior-posterior axis of the MTL become further apparent when investigating connectivity within the MTL. One study found a cross-sectional age-related increase in within-MTL connectivity (Salami et al., 2014), which later longitudinal work suggested shows a distinct pattern for anterior and posterior regions: while posterior MTL connectivity increases over time, anterior MTL shows an increase up to approximately age 55 , followed by a decrease. In contrast, another cross-sectional study found reduced connectivity with older age within the hippocampus of each hemisphere, as well as between left and right posterior hippocampus, but not anterior hippocampus (Damoiseaux et al., 2016).

While a large amount of aging research is focused on the DMN, there are other relevant networks as well. One of the major contributions of functional connectivity research to cognitive neuroscience has been the introduction of 'resting-state networks', which represent 
large-scale distributed functional systems (Smith et al., 2009). Numerous methods have been introduced to extract networks from resting-state fMRI activity, but the most prominent are independent component analysis (Beckmann et al., 2005), clustering approaches (e.g., Bellec et al., 2006; Yeo et al., 2011), and numerous other methods for delineating distinct networks (e.g., Power et al., 2011). All these methods decompose temporal fluctuations in brain activity into a set of distinct components, and while they differ in the specific analytic approaches, the extracted networks are highly consistent and reliable across methodologies (Shehzad et al., 2009; Zuo \& Xing, 2014).

The resting-state networks that are of particular interest to aging research are predominantly located within association cortex, and beside the DMN include frontoparietal control network, dorsal attention network, and salience network (see Figure 1). In general, these networks also show age-related lower functional connectivity. It has to be noted that the investigation of these networks produced a more mixed pattern of results than work concerning the DMN. The frontoparietal control network appears to have lower connectivity in old than in young subjects (Betzel et al., 2014; Fjell et al., 2015; Geerligs, Renken, et al., 2015; Grady et al., 2016). Similar results were obtained for the salience network (Betzel et al., 2014; Fjell et al., 2015). The dorsal attention network shows age-related lower connectivity in some studies (Andrews-Hanna et al., 2007), but not in others (Betzel et al., 2014).

The scarcely available work investigating within-person change in these networks also gives mixed results. $\mathrm{Ng}$ et al. (2016) found a significant decline in connectivity over time in the frontoparietal control network, but no significant change was found for the salience network. Fjell et al. (2015), while showing lower connectivity in the older group as compared to the young group for dorsal attention, salience and control networks, found a more positive change over time in the older group for the salience and the frontoparietal control network. 
Having established that functional connectivity generally decreases with age within higher-order networks, the question arises, how does the interaction between these networks change?

\section{Between-network connectivity}

Initially, most aging studies investigated how the connectivity within specific functional networks is altered with advancing age. However, as the field progressed, more studies started to investigate connectivity between different networks. Based on the literature of task-related $\mathrm{fMRI}$ studies on aging, there are several reasons to expect that between-network connectivity is especially affected by aging. One of the recurrent findings in this literature is that older adults tend to show less functional specificity in the brain regions that are engaged during the performance of a task (Park et al., 2004; Rajah \& D'Esposito, 2005). This could be a sign of neural reorganization, which might also be reflected in the functional organization of networks (Goh, 2011).

Based on these findings it would be expected that older adults also show stronger connectivity (or weaker 'negative' connectivity, known as 'anticorrelation') between distinct functional networks that are involved in different cognitive processes. Indeed this is the pattern that has been observed in many studies. One of the first studies to observe a distinction between the effects of age on within- and between-network connectivity was the work by Geerligs et al. (2014). They used a seed-based connectivity approach to investigate how connectivity between specific seed-regions and the rest of the brain was altered in older age while participants performed an oddball task. In line with previous studies, the results showed that older adults tended to show lower connectivity between the seed regions and other brain regions that were part of the same functional network.

However, higher connectivity was observed between the seeds and brain regions that were part of a different functional network. For example, they found age-related connectivity increases between the DMN, the somatosensory and the frontoparietal control network, as 
well as stronger connectivity between the frontoparietal control network and the dorsal attention network. Other studies have replicated this pattern of results in resting state data using different approaches (Betzel et al., 2014; Chan, Park, Savalia, Petersen, \& Wig, 2014; Ferreira et al., 2016; Geerligs, Renken, et al., 2015). For example, Betzel et al. (2014) investigated connectivity between a predefined set of ROls (regions of interest) and observed that ROls that had previously been associated with the same network showed reductions in connectivity with advancing age, while ROls associated with different networks mostly showed increased connectivity. This is one of many studies that have investigated connectivity within and between networks with advancing age. Unfortunately, due to differing methodological approaches, differing sets of network definition and regions of interest and differences in focus, it is difficult to pinpoint exactly which networks reliably show higher between-network connectivity in older adults. However, for some pairs of networks age-related connectivity differences are observed reliably. The dorsal attention network and the DMN are two networks that tend to show particularly strong negative connectivity or anticorrelations. Potentially due to their engagement in externally directed (dorsal attention network) and internally directed (DMN) cognition. Spreng et al. (2016) showed that the anticorrelations between these networks are reduced in older compared to younger adults, in both task and resting state. Other studies have observed similar patterns of reduced anticorrelations with advancing age (Ferreira et al., 2016; Geerligs, Renken, et al., 2015).

This increased connectivity between different networks can also be thought of as a decrease in segregation. Hence, the presented studies indicate that segregation of networks at a young age decreases with age, rendering specialized networks less specialized, with potentially detrimental effects on information processing. Indeed, Grady and colleagues (Grady et al., 2016) looked at interactions between the DMN, dorsal attention and fronto-parietal networks and they observed that an index of segregation, the within-network connectivity versus the between-network connectivity, was lower in older compared to 
younger adults in all three networks during task performance and only in the DMN and fronto-parietal networks during resting state. Using this same segregation index, Chan et al. (2014) showed that aging is associated with decreased segregation of both association cortex and cortex involved primarily in sensory-motor processing. However, their patterns of age-related differences in segregation differ, while sensory-motor systems showed a linear pattern of age-related decline, quadratic decline was observed in association systems. One concern while interpreting these results is that other factors that occur more frequently in older age, such as cognitive deficits or mild cognitive impairment, may impact the observed effects of aging on connectivity. To this end, Ferreira et al. (2016) investigated a sample which was carefully screened for cognitive deficits and lifetime psychiatric disorders. They observed very similar increases in between-network connectivity as previous studies, including as a widespread increase in connectivity between different networks with a focal loss of anticorrelations, involving mainly the connections between the DMN and the attentional networks.

The studies on between-network connectivity described so far all involved cross-sectional samples. A study by $\mathrm{Ng}$ et al. (2016) investigated connectivity between networks in a longitudinal sample. They observed that the change in connectivity between the DMN and the frontoparietal control network depends on age. While this between-network connectivity declines in participants between 60 and 70 , it increases in participants older than 75 . This finding indicates diminished segregation of networks may be a hallmark of aging.

\section{Whole-brain connectivity}

In keeping with the tradition of cognitive neuroscience, network-based analyses focus on characterizing differences in connectivity on the level of functional systems. In contrast, a whole-brain approach aims to characterize the connectivity of the entire brain, by modeling it as a complex graph of connections. The whole-brain approach, therefore, enables to 
characterize the general, brain-wide organization of functional connectivity. The characterization of the whole-brain network of functional connectivity is called the 'functional connectome'.

Graph theory, a branch of mathematics, serves as a framework to perform these investigations. In graph theory terms, a graph consists of nodes and edges. The functional connectome is reconstructed by, first, parcellating the brain into distinct regions (nodes), and second, for each pair of regions, calculating the functional connectivity between regions (edges). This results in a connectivity matrix, where each cell represents the functional connectivity between two brain regions. Based on this matrix, a wide array of graph theory metrics can be derived (Bullmore \& Sporns, 2009).

Some of these metrics allow describing properties of functional segregation, e.g. the strong connections within a module. A metric that has been investigated extensively in the aging field is local efficiency. It describes how well a node's neighbors are connected. Local efficiency is high if a node's neighbors are well-connected, and, therefore, local information flow is not heavily impeded by the loss of a node.

A consistent finding across many studies is that local efficiency is lower in older, as compared to young or middle-aged adults (Achard \& Bullmore, 2007; Geerligs, Renken, et al., 2015; Sala-Llonch et al., 2014; Song et al., 2014). By investigating a large sample that spans nearly the entire lifespan, another study found a similar, but more complex, pattern of age effects on local efficiency (Cao et al., 2014). This study found that local efficiency shows a nonlinear relationship with age, in the form of an inverted U-shape. Cross-sectionally, local efficiency increases until the age of 30 and decreases in higher age. As local efficiency is conceptually similar to within-network connectivity, these results are well in line with the findings of decreased within-network connectivity with age.

The whole-brain approach, furthermore, allows to focus on regions that have an important role by enabling information flow between modules. These so-called hub regions are 
well-connected within modules, and also have strong connections to other hubs. Hence, hubs form a so-called 'rich club' that allows to distribute and integrate information across the entire brain. Since this organization only becomes apparent when describing brain connectivity on the whole-brain level, the whole-brain approach allows for a better description of its integrative organization.

One metric that captures this organization is the 'rich-club coefficient'. The rich-club coefficient is conceptually similar to local efficiency, but instead of local connections investigates the connections between hubs. In their investigation, Cao et al. (2014) identified hubs based on functional connectivity. They found them to be located mainly in regions of the default mode, attention and visual networks. Investigating the connectivity between hub regions, they found the rich-club coefficient to follow an inverted U-shape along the lifespan. After increasing from childhood, it peaks at approximately 40 years and declines with older age. This finding indicates that global integration becomes worse with older age.

While the findings of decreasing local efficiency with age are fairly consistent, the complementary metric of global efficiency, which characterizes global parallel information flow, shows more mixed results. Many studies did not find age-correlations or differences in global efficiency (Cao et al., 2014; Geerligs, Renken, et al., 2015; Song et al., 2014), while some studies do (Achard \& Bullmore, 2007; Sala-Llonch et al., 2014). It might be that parallel information flow is not diminished with age on a global scale, but that this process is confined to certain networks, which might explain conflicting findings. Future studies will need to shed more light on this issue.

Up to now, we described work regarding functional connectivity as static. However, an interesting question is not only how regions functionally connect, but also how this connectivity changes dynamically within seconds or minutes and how these dynamics change with aging. 


\section{Dynamics}

Functional connectivity is typically estimated using the time series over the course of the whole fMRI scan. However, computing connectivity this way may disregard informative fluctuations in connectivity over the duration of the scan. Indeed, recent work has shown that even in task-free, resting-state, functional connections change over the course of a scan (Allen et al., 2014; Chang \& Glover, 2010). The first studies have now begun to investigate how aging affects these dynamics of functional connectivity. Some of these studies point to a loss of dynamics in the aging brain. Schaefer et al. (2014) looked at hubs and how they varied their participation in different networks over time. They showed reduced whole brain variation in network participation with age. Chen et al. (2017) similarly showed reduced variation of connectivity in older compared to younger adults, particularly in connections between different networks. Several studies investigated age-related dynamic connectivity differences by grouping connectivity profiles of short windows into distinct whole-brain connectivity states (Allen et al., 2014). In line with previous findings one study observed that older adults had a reduced rate of switching between distinct connectivity states (Xia et al., 2019). However, other studies observed no difference across the lifespan in the rate of switching between connectivity states (Viviano, Raz, Yuan, \& Damoiseaux, 2017). Madhyastha and Grabowski (2014) found that older adult showed more variability of connectivity within the DMN than young adults. The flexibility of connectivity in different brain regions was investigated by Yin et al. (2016), who showed that flexibility of the right middle frontal gyrus was lower in older adults compared to younger adults, with the opposite occurring for the left supramarginal gyrus. So while some studies suggest a loss of dynamics and flexibility with age, other studies show no change or even higher dynamics in older adults.

Xia et al. (2019) also investigated the occurrence of specific connectivity states in relation to age. They found that 4 out of the 5 detected states changes their occurrence with age. 
Two states with either weak connectivity between networks or anticorrelations occurred more frequently in older adults. States with strong connectivity, particularly within the salience network and the visual network occurred more frequently in older adults. Similarly, Viviano et al. (2017) found two connectivity states that occurred more frequently in older than younger adults, these were a connectivity state which was similar to the average (regular) functional connectivity profile and a state with high default mode - ventral medial temporal lobe connectivity. In addition, a state with low default mode - ventral medial temporal lobe connectivity occurred less frequency in older than younger adults. In both studies, the changes in the occurrence of dynamic connectivity states mirrored the observed age-related differences in the average connectivity profiles. These results suggest that differences in the occurrence of specific connectivity states may explain some of the age-related changes in functional connectivity that have been observed previously.

The lack of consistency between studies so far may be associated with differences in methods used and the effects of confounds. Because dynamic connectivity estimates are based on shorter time segments they are more sensitive to non-neural factors such as head motion, vascular signals and respiratory signals or other sources of noise (Chang \& Glover, 2010; Laumann et al., 2017). Group differences in dynamic connectivity may also be biased by autocorrelation and mean connectivity strength (Lehmann, White, Henson, Cam-Can, \& Geerligs, 2017). In the case of estimating connectivity states, the choice of settings (such as the number of states to estimate) can bias the results (Lehmann et al., 2017). Therefore, future studies are needed to confirm the robustness and reliability of these findings and to investigate whether considering connectivity dynamics really aids in our understanding of the mechanisms of age-related change.

\section{Cognition and functional connectivity}

Having established that functional connectivity changes with age, the question arises whether these changes are related to cognitive functioning. Most studies investigating this 
link focus on the cognitive domains of memory and processing speed in relation to functional connectivity of the DMN.

In cross-sectional studies, it has been shown that participants with higher DMN connectivity show better memory performance. This has been demonstrated for the connection between the frontal and posterior midline DMN regions (Andrews-Hanna et al., 2007), and the connection between the hippocampus and anterior DMN (Salami et al., 2014) or between hippocampus and posterior midline DMN regions (Salami et al., 2014; Wang et al., 2010). Interestingly, higher connectivity within the hippocampus seems to be related to worse memory performance (Salami et al., 2014). However, other studies, while finding age-related lower memory performance in old participants, did not find a link between memory and functional connectivity within the DMN (Damoiseaux et al., 2008) or the hippocampus (Damoiseaux et al., 2016). Looking beyond the DMN, it has been shown that higher functional specialization of different networks is associated with lower episodic memory performance (Chan et al., 2014). This was measured using segregation, an index of within- versus between-network connectivity. Interestingly this association between memory and segregation was particularly present for networks within association cortex (including the DMN, frontoparietal network, and dorsal attention network), and not for networks involved in primary sensorimotor functions.

In contrast to cross-sectional study designs, longitudinal designs provide stronger evidence for brain-cognition links in aging that are not impeded by cohort effects (see Limitations section). Studies that simultaneously tracked changes in functional connectivity and memory have found steeper decline in within-DMN connectivity to be related to steeper decline in episodic memory performance (Fjell et al., 2015; Persson et al., 2014; Staffaroni et al., 2018). More generally, this pattern can also be found for the connectivity seeded from hippocampus and basal ganglia regions, respectively, to widespread cortical regions: change in memory is positively related to change in functional connectivity (Fjell et al., 2016). 
Furthermore, changes in connectivity in the left posterior MTL also show a relationship to changes in memory scores, mirroring cross-sectional patterns in the hippocampus: greater functional connectivity increases go together with a steeper decline in memory (Salami et al., 2016).

Other studies track memory performance longitudinally and then investigate functional connectivity at the end of the observation interval. This approach allows for contrasting functional connectivity between subjects with declining and stable memory. Functional connectivity in regions of the DMN has been found to be lower in participants with declining memory than in participants with stable memory (Bernard et al., 2015). Furthermore, the reverse pattern can be seen for connectivity within the hippocampus: older adults with stable memory showed lower connectivity within the hippocampus, a pattern that makes them more similar to young adults (Salami et al., 2014).

Cross-sectional studies investigating associations between processing speed and functional connectivity indicate that lower DMN connectivity is associated with slower processing speed in older adults (Andrews-Hanna et al., 2007; Damoiseaux et al., 2008; Hirsiger et al., 2016). However, connectivity of the hippocampus with DMN regions did not reveal a relationship to processing speed performance in an adult lifespan sample (Damoiseaux et al., 2016). A previous longitudinal study found a relationship between steeper processing speed declines and within-DMN connectivity (Staffaroni et al., 2018), however, another study did not find this link ( $\mathrm{Ng}$ et al., 2016). The authors of the latter study also investigated between-network connectivity and found that a steeper decline in processing speed was associated with a steeper decline in between-network segregation between the DMN and the frontoparietal control network ( $\mathrm{Ng}$ et al., 2016).

One mechanism to explain these conflicting results regarding the link between functional connectivity and cognitive functioning in healthy aging might be compensatory neuronal processes. According to the revised Scaffolding Theory of Aging and Cognition (STAC-r) 
(Reuter-Lorenz \& Park, 2014), adversary changes in functional connectivity might be counteracted by compensatory processes, which might weaken or delay the effect the of adversary brain changes on cognitive decline. Therefore, simultaneously describing brain and cognitive changes might miss lagged relationships that can only be seen later in life, when compensatory mechanisms break down. Investigating lagged relationships, however, requires research designs with at least three measurement occasions over multiple years, and while there exist very few studies doing that, they have not yet investigated lagged relationships. In neurodegenerative disorders, however, compensatory processes might no longer be able to counteract changes in brain connectivity, hence, changes in connectivity might more directly result in cognitive decline.

\section{Functional connectivity in neurodegenerative disorders}

So far we have mainly discussed changes in functional connectivity observed in typical aging, but how does functional brain connectivity change in age-related neurodegenerative disorders such as Alzheimer's Disease $(A D)$ ? The existing literature indicates that particularly the DMN, which has consistently shown lower functional connectivity with older age, seems further affected by $A D$. Most cross-sectional work indicates lower DMN connectivity in clinically diagnosed $A D$ patients compared to cognitively unimpaired older adults (Badhwar et al., 2017; Binnewijzend et al., 2012; Greicius, Srivastava, Reiss, \& Menon, 2004). However, longitudinal work suggests an initial increase of DMN functional connectivity that is later followed by a decrease in the same areas (Bai et al., 2011; Damoiseaux, Prater, Miller, \& Greicius, 2012). These longitudinal studies furthermore suggest that areas within the DMN are affected relatively early at the Mild Cognitive Impairment $(\mathrm{MCl})$ and early dementia stage, with additional brain networks affected as the disease progresses. The observed initial increase in connectivity may reflect a compensatory process, as outlined in the revised STAC-r model, a model that encompasses both typical and pathological aging (Reuter-Lorenz \& Park, 2014). The STAC-r model poses 
that harmful effects of functional and structural brain changes on cognition can be muted by "compensatory scaffolding," such as recruitment of more neural circuitry. This compensatory process functions as an attempt to maintain typical cognition in response to numerous typical and pathological age-related neural insults. In AD this compensation process starting in the posterior $\mathrm{DMN}$ can also be viewed as a transient load-shifting process, which eventually will result in a cascading network failure, as described by (Jones et al., 2016).

The important role of the DMN in AD is in line with the DMN's involvement in internally focused activities. Activities such as self-referential thinking and prospective memory encompass the same brain regions that are active during successfully retrieving memories, i.e. more brain activity in response to old items correctly identified as old compared to new items identified as new (Buckner, Andrews-Hanna, \& Schacter, 2008). Furthermore, the DMN overlaps with observed brain patterns of gray matter volume loss and amyloid- $\beta$ deposition in AD (Buckner et al., 2008, 2005). Deficits in memory retrieval, gray matter atrophy and accumulation of amyloid- $\beta$ are all established indicators for AD. More specifically, the presence of amyloid- $\beta$ aggregations (plaques) in the brain is one of the defining pathological markers of $\mathrm{AD}$, along with tau pathology (neurofibrillary tangles). The observed overlap of these $A D$ markers to the $D M N$, and the observed lower functional connectivity in $A D$ patients, indicates specific vulnerability of these brain regions to $A D$ pathology.

Even though $\mathrm{AD}$ is the most common neurodegenerative disorder, it is not the only one. Since other neurodegenerative disorders present with different cognitive/behavioral deficits, do they also affect different brain networks? Seeley and colleagues (2009) addressed this question and found that specific brain networks have selective vulnerability to specific neurodegenerative disorders. In line with previous research they observed grey matter atrophy within the $\mathrm{DMN}$ in $\mathrm{AD}$, but in addition they observed different grey matter atrophy patterns in other neurodegenerative disorders. For example, in behavioral variant 
frontotemporal dementia the atrophy pattern overlapped with the salience network, and in progressive nonfluent aphasia it converged with the frontoparietal network (Seeley et al., 2009). In subsequent research they examined functional connectivity differences and observed divergent functional connectivity between behavioral variant frontotemporal dementia and $A D$, such that the former had lower salience network and higher DMN connectivity whereas for the latter the opposite was true (Zhou et al., 2010), an effect that was later replicated in an EEG study (Yu et al., 2016).

\section{Functional connectivity as a biomarker}

The evidence showing age effects on functional connectivity supports its potential application as a biomarker that might allow for predictions on an individual level. For instance, the "brain-age prediction" approach aims to provide an aggregated marker of brain health. Furthermore, functional connectivity seems to be affected early on in the course of neurodegenerative disorders, long before neuropathology manifests itself in cognitive decline (G. Chen et al., 2016). Therefore, connectivity might be useful in predicting future disease development.

\section{Functional connectivity as a general biomarker for brain health}

Aging results in changes in brain function as well as in brain structure. Developing a biomarker for general brain health requires reducing the dimensionality of this high-dimensional pattern so that multiple three-dimensional brain maps are distilled into a small number of numeric values that allow for a more straightforward interpretation of brain aging. This biomarker can then be used to identify individuals with accelerated brain aging, or serve as an outcome measure in intervention studies. Machine learning methods have been used to try to identify such biomarkers. For example, a single person's biological brain age can be estimated based on neuroimaging data and compared to their chronological age. This means that if a person is 50 years old and their brain looks like the brain of a typical 70 
year old, this can be taken as evidence for increased brain aging. The brain-age approach has been applied to a variety of neuro-cognitive and psychiatric disorders and could demonstrate that these patients have older appearing brains than typical individuals. Importantly, this approach can also be used to investigate protective effects of lifestyle on brain aging (Cole \& Franke, 2017).

The majority of extant studies that investigated brain aging relied on structural brain data, measuring, for instance, the amount of volume loss in the cortex and subcortical regions. More recent work extended this approach to include functional connectivity data (Liem et al., 2017). The combination of structural and functional connectivity data increased the prediction accuracy of the models and could therefore more fully characterize brain aging. This finding was validated further by showing that participants with mild or major cognitive impairment show progressively increasing functional and structural brain aging scores, indicating that accelerated brain aging is related to accelerated deterioration in cognitive functioning.

\section{Connectivity as an early marker for neurodegenerative disorders}

It is assumed that the neurodegenerative process starts years, perhaps even decades, before measurable cognitive impairment. Therefore, an effort is made to detect neurodegenerative brain changes at an earlier stage by examining cognitively unimpaired older adults with heightened risk for dementia or AD, such as presence of genetic risk (e.g. APOE- $\varepsilon 4$ carriers), neuropathology (e.g. amyloid- $\beta$ and tau deposition in the brain), or subjective cognitive decline (i.e. a subjective concern of cognitive decline in the absence of objective impairment). Researchers have compared DMN connectivity in APOE-£4 carriers, which is the most prominent genetic risk factor for late-onset $A D$, to those with ع3-homozygotes, the neutral risk alleles. They found that participants with an at-risk genotype had lower DMN functional connectivity than the neutral risk group, even though they were not cognitively impaired (Machulda et al., 2011; Sheline et al., 2010). A 
longitudinal study in cognitively unimpaired older adults found that the at-risk group (i.e. APOE- $\varepsilon 4$ carriers) had more rapid loss of functional segregation between the DMN and the executive control network over time than the neutral risk group ( $\mathrm{Ng}$ et al., 2018). These studies suggest that altered within and between DMN connectivity is an indicator of AD risk. Note that the APOE-£4 effect on DMN functional connectivity may be specific to women (Damoiseaux, Seeley, et al., 2012; Heise et al., 2014), which is in line with other research showing higher incidence of $A D$, faster disease progression and more neurodegenerative brain changes in women compared to men (Lin et al., 2015; Mielke, Vemuri, \& Rocca, 2014). Lower DMN connectivity was also observed in cognitively unimpaired adults considered to have preclinical $A D$, i.e. who have pathologic amyloid- $\beta+/$ - tau biomarkers (Jones et al., 2017; Mormino et al., 2011), and in individuals with subjective cognitive decline (Viviano et al., 2019). These studies indicate the potential for functional connectivity, mainly of the DMN, as a marker for early neurodegenerative disease detection and progression.

\section{Methodological considerations relevant to aging research}

Cross-sectional and longitudinal study designs allow to investigate different effects. It is important to distinguish effects of age-related differences (between-person effects) from effects of age-related change (within-person effects). The majority of aging neuroscience studies use cross-sectional designs, sampling participants over a wide age range or young and old groups at one measurement occasion. However, interpreting these differences as a proxy for within-person changes is problematic, as, amongst other issues, these designs are confounded by cohort effects. For instance, since the nutritional and educational environment changed over the last decades, differences between a 30 and a 80 year old participant will not only reflect within-person changes over time, but also differences due to different environments in which the participants grew up. Within-person change can only be studied with longitudinal research designs, which have demonstrated different results as compared to cross-sectional studies (Pfefferbaum \& Sullivan, 2015). However, since 
longitudinal studies are much more time- and resource intensive than cross-sectional studies, the majority of studies rely on cross-sectional designs.

Computing functional connectivity involves choices between analysis approaches (e.g. correlation based approaches versus independent component analysis), choices about which networks or regions of interest to focus on and choices about how to pre-process the data. Each of these choices can have a major impact on the results of a study. The studies described in this chapter vary widely in the approaches that are used. The effects of different analysis choices and pre-processing steps on age-related differences in connectivity were investigated in a recent study (Geerligs, Tsvetanov, Cam-Can, \& Henson, 2017). They found that different preprocessing options can substantially alter the observed association between age and functional connectivity. In part, this is because fMRI data contains signals that do not have a neural origin. Some of these signals reflect head motion of participants in the scanner (Power, Barnes, Snyder, Schlaggar, \& Petersen, 2012). Even small amounts of head motion can significantly affect the fMRI data and these effects are very difficult to remove completely, even with very elaborate pre-processing pipelines (Power et al., 2014; Van Dijk, Sabuncu, \& Buckner, 2012). In the context of aging, this is particularly concerning because older adults tend to move more when they are in the scanner compared to younger adults (D’Esposito, Zarahn, Aguirre, \& Rypma, 1999; Geerligs, Rubinov, Cam-Can, \& Henson, 2015). Another factor that is important in the context of aging is the impact of breathing and heart rate variability on the fMRI signal. Recent work has shown that these can introduce substantial changes in the fMRI signal, and that these changes are shared between regions, thereby inducing apparent connectivity without any neural origin (Power, Plitt, Laumann, \& Martin, 2017). Heart rate variability also decreases with age, and these changes can confound the observed associations between age and connectivity (Geerligs et al., 2017). These effects can be mitigated by specific pre- and post-processing steps, but those are not commonplace yet. 
Studies also vary in the networks or regions they focus on, which makes them difficult to compare. Even studies focusing on the same networks can use different regions of interest (ROIs). This is potentially important because misalignment of ROls with true functional regions will cause greater heterogeneity in the signals within ROls, which may lead to weakened functional connectivity estimates and seemingly reduced segregation between brain systems (Geerligs et al., 2017). The location or number of true functional regions may change with age, causing misalignment with the ROls used for analysis.

Given these confounds and limitations it is important to ensure that any conclusions that are drawn hold across a range of analysis choices and datasets. That is why it is encouraging to see that there are patterns of age-related differences that are observed consistently across studies, such as the consistent decline in connectivity within the DMN and the loss of network segregation with advancing age.

\section{Summary and future directions}

Aging has pronounced effects on the brain's functional connectivity architecture. Connectivity within the DMN, as well as in other association networks decreases with older age, while the connectivity between different functional systems increases. Together, these changes result in a loss of segregation between different functional systems. Nevertheless, findings regarding the association between connectivity and cognition remain sparse and unclear. This may be due to the limitations of the cross-sectional nature of most studies in the field, as well as to compensatory processes that older adults may engage to maintain higher levels of cognitive abilities. Future studies using longitudinal research designs, tracking study participants across several years and recording data about their brain and cognitive status, are imperative to further this area of research.

The field has largely advanced alongside the methodological innovation in data acquisition and analysis. Continuing innovation in describing brain connectivity will provide novel insights into the aging brain in the future. Large, multi-site datasets will help to 
disambiguate contradictory results from previous studies that often based their findings on small datasets, which might produce non-replicable results.

\section{Acknowledgements}

FL was supported by the University Research Priority Program "Dynamics of Healthy Aging" at the University of Zurich. LG was supported by a Veni grant [451-16-013] from the Netherlands Organization for Scientific Research.

\section{$\underline{\text { References }}$}

Achard, S., \& Bullmore, E. (2007). Efficiency and cost of economical brain functional networks. PLoS Computational Biology, 3(2), e17.

Allen, E. A., Damaraju, E., Plis, S. M., Erhardt, E. B., Eichele, T., \& Calhoun, V. D. (2014). Tracking whole-brain connectivity dynamics in the resting state. Cerebral Cortex , 24(3), 663-676.

Andrews-Hanna, J. R., Smallwood, J., \& Spreng, R. N. (2014). The default network and self-generated thought: component processes, dynamic control, and clinical relevance. Annals of the New York Academy of Sciences, 1316, 29-52.

Andrews-Hanna, J. R., Snyder, A. Z., Vincent, J. L., Lustig, C., Head, D., Raichle, M. E., \& Buckner, R. L. (2007). Disruption of large-scale brain systems in advanced aging. Neuron, 56(5), 924-935.

Badhwar, A., Tam, A., Dansereau, C., Orban, P., Hoffstaedter, F., \& Bellec, P. (2017). Resting-state network dysfunction in Alzheimer's disease: A systematic review and meta-analysis. Alzheimer's \& Dementia: The Journal of the Alzheimer's Association, 8, 73-85.

Bai, F., Watson, D. R., Shi, Y., Wang, Y., Yue, C., YuhuanTeng, ... Zhang, Z. (2011). Specifically progressive deficits of brain functional marker in amnestic type mild 
cognitive impairment. PloS One, 6(9), e24271.

Beckmann, C. F., DeLuca, M., Devlin, J. T., \& Smith, S. M. (2005). Investigations into resting-state connectivity using independent component analysis. Philosophical Transactions of the Royal Society of London. Series B, Biological Sciences, 360(1457), $1001-1013$.

Bellec, P., Perlbarg, V., Jbabdi, S., Pélégrini-Issac, M., Anton, J.-L., Doyon, J., \& Benali, H. (2006). Identification of large-scale networks in the brain using fMRI. Neurolmage, 29(4), 1231-1243.

Bernard, C., Dilharreguy, B., Helmer, C., Chanraud, S., Amieva, H., Dartigues, J.-F., ... Catheline, G. (2015). PCC characteristics at rest in 10-year memory decliners. Neurobiology of Aging, 36(10), 2812-2820.

Betzel, R. F., Byrge, L., He, Y., Goñi, J., Zuo, X.-N., \& Sporns, O. (2014). Changes in structural and functional connectivity among resting-state networks across the human lifespan. Neurolmage, 102 Pt 2, 345-357.

Binnewijzend, M. A. A., Schoonheim, M. M., Sanz-Arigita, E., Wink, A. M., van der Flier, W. M., Tolboom, N., ... Barkhof, F. (2012). Resting-state fMRI changes in Alzheimer's disease and mild cognitive impairment. Neurobiology of Aging, 33(9), 2018-2028.

Biswal, B., Yetkin, F. Z., Haughton, V. M., \& Hyde, J. S. (1995). Functional connectivity in the motor cortex of resting human brain using echo-planar MRI. Magnetic Resonance in Medicine: Official Journal of the Society of Magnetic Resonance in Medicine / Society of Magnetic Resonance in Medicine, 34(4), 537-541.

Buckner, R. L., Andrews-Hanna, J. R., \& Schacter, D. L. (2008). The brain's default network: anatomy, function, and relevance to disease. Annals of the New York Academy of Sciences, 1124, 1-38.

Buckner, R. L., Snyder, A. Z., Shannon, B. J., LaRossa, G., Sachs, R., Fotenos, A. F., ... Mintun, M. A. (2005). Molecular, structural, and functional characterization of 
Alzheimer's disease: evidence for a relationship between default activity, amyloid, and memory. The Journal of Neuroscience: The Official Journal of the Society for Neuroscience, 25(34), 7709-7717.

Bullmore, E., \& Sporns, O. (2009). Complex brain networks: graph theoretical analysis of structural and functional systems. Nature Reviews. Neuroscience, 10(3), 186-198.

Cao, M., Wang, J.-H., Dai, Z.-J., Cao, X.-Y., Jiang, L.-L., Fan, F.-M., ... He, Y. (2014). Topological organization of the human brain functional connectome across the lifespan. Developmental Cognitive Neuroscience, 7, 76-93.

Chang, C., \& Glover, G. H. (2010). Time-frequency dynamics of resting-state brain connectivity measured with fMRI. Neurolmage, 50(1), 81-98.

Chan, M. Y., Park, D. C., Savalia, N. K., Petersen, S. E., \& Wig, G. S. (2014). Decreased segregation of brain systems across the healthy adult lifespan. Proceedings of the National Academy of Sciences of the United States of America, 111(46), E4997-E5006.

Chen, G., Shu, H., Chen, G., Ward, B. D., Antuono, P. G., Zhang, Z., ... Alzheimer's Disease Neuroimaging Initiative. (2016). Staging Alzheimer's Disease Risk by Sequencing Brain Function and Structure, Cerebrospinal Fluid, and Cognition Biomarkers. Journal of Alzheimer's Disease: JAD, 54(3), 983-993.

Chen, Y., Wang, W., Zhao, X., Sha, M., Liu, Y. 'nan, Zhang, X., ... Ming, D. (2017).

Age-Related Decline in the Variation of Dynamic Functional Connectivity: A Resting State Analysis. Frontiers in Aging Neuroscience, 9, 203.

Cole, J. H., \& Franke, K. (2017). Predicting Age Using Neuroimaging: Innovative Brain Ageing Biomarkers. Trends in Neurosciences, 40(12), 681-690.

Damoiseaux, J. S., Beckmann, C. F., Arigita, E. J. S., Barkhof, F., Scheltens, P., Stam, C. J., ... Rombouts, S. A. R. B. (2008). Reduced resting-state brain activity in the "default network" in normal aging. Cerebral Cortex , 18(8), 1856-1864.

Damoiseaux, J. S., Prater, K. E., Miller, B. L., \& Greicius, M. D. (2012). Functional 
connectivity tracks clinical deterioration in Alzheimer's disease. Neurobiology of Aging, 33(4), 828.e19-e30.

Damoiseaux, J. S., S A R, Barkhof, F., Scheltens, P., Stam, C. J., Smith, S. M., \& Beckmann, C. F. (2006). Consistent resting-state networks across healthy subjects. Proceedings of the National Academy of Sciences, 103(37), 13848-13853.

Damoiseaux, J. S., Seeley, W. W., Zhou, J., Shirer, W. R., Coppola, G., Karydas, A., ... Alzheimer's Disease Neuroimaging Initiative. (2012). Gender modulates the APOE $\varepsilon 4$ effect in healthy older adults: convergent evidence from functional brain connectivity and spinal fluid tau levels. The Journal of Neuroscience: The Official Journal of the Society for Neuroscience, 32(24), 8254-8262.

Damoiseaux, J. S., Viviano, R. P., Yuan, P., \& Raz, N. (2016). Differential effect of age on posterior and anterior hippocampal functional connectivity. Neurolmage, 133, 468-476.

Daselaar, S. M., Prince, S. E., Dennis, N. A., Hayes, S. M., Kim, H., \& Cabeza, R. (2009). Posterior midline and ventral parietal activity is associated with retrieval success and encoding failure. Frontiers in Human Neuroscience, 3, 13.

De Luca, M., Beckmann, C. F., De Stefano, N., Matthews, P. M., \& Smith, S. M. (2006). fMRI resting state networks define distinct modes of long-distance interactions in the human brain. Neurolmage, 29(4), 1359-1367.

D’Esposito, M., Zarahn, E., Aguirre, G. K., \& Rypma, B. (1999). The effect of normal aging on the coupling of neural activity to the bold hemodynamic response. Neurolmage, 10(1), 6-14.

Ferreira, L. K., Regina, A. C. B., Kovacevic, N., Martin, M. da G. M., Santos, P. P., Carneiro, C. de G., ... Busatto, G. F. (2016). Aging Effects on Whole-Brain Functional Connectivity in Adults Free of Cognitive and Psychiatric Disorders. Cerebral Cortex, 26(9), 3851-3865.

Fjell, A. M., Sneve, M. H., Grydeland, H., Storsve, A. B., de Lange, A.-M. G., Amlien, I. K., ... 
Walhovd, K. B. (2015). Functional connectivity change across multiple cortical networks relates to episodic memory changes in aging. Neurobiology of Aging, 36(12), 3255-3268.

Fjell, A. M., Sneve, M. H., Storsve, A. B., Grydeland, H., Yendiki, A., \& Walhovd, K. B. (2016). Brain Events Underlying Episodic Memory Changes in Aging: A Longitudinal Investigation of Structural and Functional Connectivity. Cerebral Cortex , 26(3), $1272-1286$.

Fraser, M. A., Shaw, M. E., \& Cherbuin, N. (2015). A systematic review and meta-analysis of longitudinal hippocampal atrophy in healthy human ageing. Neurolmage, 112, 364-374.

Friston, K. J. (1994). Functional and effective connectivity in neuroimaging: A synthesis. Human Brain Mapping, 2(1-2), 56-78.

Geerligs, L., Maurits, N. M., Renken, R. J., \& Lorist, M. M. (2014). Reduced specificity of functional connectivity in the aging brain during task performance. Human Brain Mapping, 35(1), 319-330.

Geerligs, L., Renken, R. J., Saliasi, E., Maurits, N. M., \& Lorist, M. M. (2015). A Brain-Wide Study of Age-Related Changes in Functional Connectivity. Cerebral Cortex , 25(7), 1987-1999.

Geerligs, L., Rubinov, M., Cam-Can, \& Henson, R. N. (2015). State and Trait Components of Functional Connectivity: Individual Differences Vary with Mental State. The Journal of Neuroscience: The Official Journal of the Society for Neuroscience, 35(41), $13949-13961$.

Geerligs, L., Tsvetanov, K. A., Cam-Can, \& Henson, R. N. (2017). Challenges in measuring individual differences in functional connectivity using fMRI: The case of healthy aging. Human Brain Mapping, 38(8), 4125-4156.

Goh, J. O. S. (2011). Functional Dedifferentiation and Altered Connectivity in Older Adults: Neural Accounts of Cognitive Aging. Aging and Disease, 2(1), 30-48. 
Grady, C., Sarraf, S., Saverino, C., \& Campbell, K. (2016). Age differences in the functional interactions among the default, frontoparietal control, and dorsal attention networks. Neurobiology of Aging, 41, 159-172.

Greicius, M. D., Kiviniemi, V., Tervonen, O., Vainionpää, V., Alahuhta, S., Reiss, A. L., \& Menon, V. (2008). Persistent default-mode network connectivity during light sedation. Human Brain Mapping, 29(7), 839-847.

Greicius, M. D., Krasnow, B., Reiss, A. L., \& Menon, V. (2003). Functional connectivity in the resting brain: A network analysis of the default mode hypothesis. Proceedings of the National Academy of Sciences of the United States of America, 100(1), 253-258.

Greicius, M. D., Srivastava, G., Reiss, A. L., \& Menon, V. (2004). Default-mode network activity distinguishes Alzheimer's disease from healthy aging: evidence from functional MRI. Proceedings of the National Academy of Sciences of the United States of America, 101(13), 4637-4642.

Heise, V., Filippini, N., Trachtenberg, A. J., Suri, S., Ebmeier, K. P., \& Mackay, C. E. (2014). Apolipoprotein E genotype, gender and age modulate connectivity of the hippocampus in healthy adults. Neurolmage, 98, 23-30.

Hirsiger, S., Koppelmans, V., Mérillat, S., Liem, F., Erdeniz, B., Seidler, R. D., \& Jäncke, L. (2016). Structural and functional connectivity in healthy aging: Associations for cognition and motor behavior. Human Brain Mapping, 37(3), 855-867.

Jones, D. T., Graff-Radford, J., Lowe, V. J., Wiste, H. J., Gunter, J. L., Senjem, M. L., ... Jack, C. R., Jr. (2017). Tau, amyloid, and cascading network failure across the Alzheimer's disease spectrum. Cortex; a Journal Devoted to the Study of the Nervous System and Behavior, 97, 143-159.

Jones, D. T., Knopman, D. S., Gunter, J. L., Graff-Radford, J., Vemuri, P., Boeve, B. F., ... Alzheimer's Disease Neuroimaging Initiative. (2016). Cascading network failure across the Alzheimer's disease spectrum. Brain: A Journal of Neurology, 139(Pt 2), 547-562. 
Laumann, T. O., Snyder, A. Z., Mitra, A., Gordon, E. M., Gratton, C., Adeyemo, B., ... Petersen, S. E. (2017). On the Stability of BOLD fMRI Correlations. Cerebral Cortex, 27(10), 4719-4732.

Lehmann, B. C. L., White, S. R., Henson, R. N., Cam-Can, \& Geerligs, L. (2017). Assessing dynamic functional connectivity in heterogeneous samples. Neurolmage, 157, 635-647.

Liem, F., Varoquaux, G., Kynast, J., Beyer, F., Kharabian Masouleh, S., Huntenburg, J. M., ... Margulies, D. S. (2017). Predicting brain-age from multimodal imaging data captures cognitive impairment. Neurolmage, 148, 179-188.

Lin, K. A., Choudhury, K. R., Rathakrishnan, B. G., Marks, D. M., Petrella, J. R., Doraiswamy, P. M., \& Alzheimer's Disease Neuroimaging Initiative. (2015). Marked gender differences in progression of mild cognitive impairment over 8 years. Alzheimer's \& Dementia: The Journal of the Alzheimer's Association, 1(2), 103-110.

Lu, H., Zou, Q., Gu, H., Raichle, M. E., Stein, E. A., \& Yang, Y. (2012). Rat brains also have a default mode network. Proceedings of the National Academy of Sciences, 109(10), 3979-3984.

Machulda, M. M., Jones, D. T., Vemuri, P., McDade, E., Avula, R., Przybelski, S., ... Jack, C. R., Jr. (2011). Effect of APOE \&4 status on intrinsic network connectivity in cognitively normal elderly subjects. Archives of Neurology, 68(9), 1131-1136.

Madhyastha, T. M., \& Grabowski, T. J. (2014). Age-related differences in the dynamic architecture of intrinsic networks. Brain Connectivity, 4(4), 231-241.

Mak, L. E., Minuzzi, L., MacQueen, G., Hall, G., Kennedy, S. H., \& Milev, R. (2017). The Default Mode Network in Healthy Individuals: A Systematic Review and Meta-Analysis. Brain Connectivity, 7(1), 25-33.

Mielke, M. M., Vemuri, P., \& Rocca, W. A. (2014). Clinical epidemiology of Alzheimer's disease: assessing sex and gender differences. Clinical Epidemiology, 6, 37-48.

Mormino, E. C., Smiljic, A., Hayenga, A. O., Onami, S. H., Greicius, M. D., Rabinovici, G. D., 
... Jagust, W. J. (2011). Relationships between $\beta$-amyloid and functional connectivity in different components of the default mode network in aging. Cerebral Cortex , 21(10), 2399-2407.

Ng, K. K., Lo, J. C., Lim, J. K. W., Chee, M. W. L., \& Zhou, J. (2016). Reduced functional segregation between the default mode network and the executive control network in healthy older adults: A longitudinal study. Neurolmage, 133, 321-330.

Ng, K. K., Qiu, Y., Lo, J. C.-Y., Koay, E. S.-C., Koh, W.-P., Chee, M. W.-L., \& Zhou, J. (2018). Functional segregation loss over time is moderated by APOE genotype in healthy elderly. Human Brain Mapping, 39(7), 2742-2752.

Park, D. C., Polk, T. A., Park, R., Minear, M., Savage, A., \& Smith, M. R. (2004). From The Cover: Aging reduces neural specialization in ventral visual cortex. Proceedings of the National Academy of Sciences, 101(35), 13091-13095.

Persson, J., Pudas, S., Nilsson, L.-G., \& Nyberg, L. (2014). Longitudinal assessment of default-mode brain function in aging. Neurobiology of Aging, 35(9), 2107-2117.

Pfefferbaum, A., \& Sullivan, E. V. (2015). Cross-sectional versus longitudinal estimates of age-related changes in the adult brain: overlaps and discrepancies. Neurobiology of Aging, 36(9), 2563-2567.

Pini, L., Pievani, M., Bocchetta, M., Altomare, D., Bosco, P., Cavedo, E., ... Frisoni, G. B. (2016). Brain atrophy in Alzheimer's Disease and aging. Ageing Research Reviews, 30, 25-48.

Power, J. D., Barnes, K. A., Snyder, A. Z., Schlaggar, B. L., \& Petersen, S. E. (2012). Spurious but systematic correlations in functional connectivity MRI networks arise from subject motion. Neurolmage, 59(3), 2142-2154.

Power, J. D., Cohen, A. L., Nelson, S. M., Wig, G. S., Barnes, K. A., Church, J. A., ... Petersen, S. E. (2011). Functional network organization of the human brain. Neuron, 72(4), 665-678. 
Power, J. D., Mitra, A., Laumann, T. O., Snyder, A. Z., Schlaggar, B. L., \& Petersen, S. E. (2014). Methods to detect, characterize, and remove motion artifact in resting state fMRI. Neurolmage, 84, 320-341.

Power, J. D., Plitt, M., Laumann, T. O., \& Martin, A. (2017). Sources and implications of whole-brain fMRI signals in humans. Neurolmage, 146, 609-625.

Raichle, M. E. (2015). The brain's default mode network. Annual Review of Neuroscience, 38, 433-447.

Raichle, M. E., MacLeod, A. M., Snyder, A. Z., Powers, W. J., Gusnard, D. A., \& Shulman, G. L. (2001). A default mode of brain function. Proceedings of the National Academy of Sciences of the United States of America, 98(2), 676-682.

Rajah, M. N., \& D’Esposito, M. (2005). Region-specific changes in prefrontal function with age: a review of PET and fMRI studies on working and episodic memory. Brain: $A$ Journal of Neurology, 128(Pt 9), 1964-1983.

Reuter-Lorenz, P. A., \& Park, D. C. (2014). How does it STAC up? Revisiting the scaffolding theory of aging and cognition. Neuropsychology Review, 24(3), 355-370.

Sala-Llonch, R., Junqué, C., Arenaza-Urquijo, E. M., Vidal-Piñeiro, D., Valls-Pedret, C., Palacios, E. M., ... Bartrés-Faz, D. (2014). Changes in whole-brain functional networks and memory performance in aging. Neurobiology of Aging, 35(10), 2193-2202.

Salami, A., Pudas, S., \& Nyberg, L. (2014). Elevated hippocampal resting-state connectivity underlies deficient neurocognitive function in aging. Proceedings of the National Academy of Sciences of the United States of America, 111(49), 17654-17659.

Salami, A., Wåhlin, A., Kaboodvand, N., Lundquist, A., \& Nyberg, L. (2016). Longitudinal Evidence for Dissociation of Anterior and Posterior MTL Resting-State Connectivity in Aging: Links to Perfusion and Memory. Cerebral Cortex , 26(10), 3953-3963.

Schaefer, A., Margulies, D. S., Lohmann, G., Gorgolewski, K. J., Smallwood, J., Kiebel, S. J., \& Villringer, A. (2014). Dynamic network participation of functional connectivity hubs 
assessed by resting-state fMRI. Frontiers in Human Neuroscience, 8, 195.

Seeley, W. W., Crawford, R. K., Zhou, J., Miller, B. L., \& Greicius, M. D. (2009). Neurodegenerative diseases target large-scale human brain networks. Neuron, 62(1), 42-52.

Shehzad, Z., Kelly, A. M. C., Reiss, P. T., Gee, D. G., Gotimer, K., Uddin, L. Q., ... Milham, M. P. (2009). The resting brain: unconstrained yet reliable. Cerebral Cortex , 19(10), 2209-2229.

Sheline, Y. I., Morris, J. C., Snyder, A. Z., Price, J. L., Yan, Z., D’Angelo, G., ... Mintun, M. A. (2010). APOE4 allele disrupts resting state fMRI connectivity in the absence of amyloid plaques or decreased CSF Aß42. The Journal of Neuroscience: The Official Journal of the Society for Neuroscience, 30(50), 17035-17040.

Shulman, G. L., Fiez, J. A., Corbetta, M., Buckner, R. L., Miezin, F. M., Raichle, M. E., \& Petersen, S. E. (1997). Common Blood Flow Changes across Visual Tasks: II. Decreases in Cerebral Cortex. Journal of Cognitive Neuroscience, 9(5), 648-663.

Smith, S. M., Fox, P. T., Miller, K. L., Glahn, D. C., Mickle Fox, P., Mackay, C. E., ... Beckmann, C. F. (2009). Correspondence of the brain's functional architecture during activation and rest. Proceedings of the National Academy of Sciences of the United States of America, 106(31), 13040-13045.

Song, J., Birn, R. M., Boly, M., Meier, T. B., Nair, V. A., Meyerand, M. E., \& Prabhakaran, V. (2014). Age-related reorganizational changes in modularity and functional connectivity of human brain networks. Brain Connectivity, 4(9), 662-676.

Spreng, R. N., Nathan Spreng, R., Dale Stevens, W., Viviano, J. D., \& Schacter, D. L. (2016). Attenuated anticorrelation between the default and dorsal attention networks with aging: evidence from task and rest. Neurobiology of Aging, 45, 149-160.

Staffaroni, A. M., Brown, J. A., Casaletto, K. B., Elahi, F. M., Deng, J., Neuhaus, J., ... Kramer, J. H. (2018). The Longitudinal Trajectory of Default Mode Network Connectivity 
in Healthy Older Adults Varies As a Function of Age and Is Associated with Changes in Episodic Memory and Processing Speed. The Journal of Neuroscience: The Official Journal of the Society for Neuroscience, 38(11), 2809-2817.

Van Dijk, K. R. A., Sabuncu, M. R., \& Buckner, R. L. (2012). The influence of head motion on intrinsic functional connectivity MRI. Neurolmage, 59(1), 431-438.

Vincent, J. L., Patel, G. H., Fox, M. D., Snyder, A. Z., Baker, J. T., Van Essen, D. C., ... Raichle, M. E. (2007). Intrinsic functional architecture in the anaesthetized monkey brain. Nature, 447(7140), 83-86.

Viviano, R. P., Hayes, J. M., Pruitt, P. J., Fernandez, Z. J., van Rooden, S., van der Grond, J., ... Damoiseaux, J. S. (2019). Aberrant memory system connectivity and working memory performance in subjective cognitive decline. Neurolmage, 185, 556-564.

Viviano, R. P., Raz, N., Yuan, P., \& Damoiseaux, J. S. (2017). Associations between dynamic functional connectivity and age, metabolic risk, and cognitive performance. Neurobiology of Aging, 59, 135-143.

Wang, L., Laviolette, P., O’Keefe, K., Putcha, D., Bakkour, A., Van Dijk, K. R. A., ... Sperling, R. A. (2010). Intrinsic connectivity between the hippocampus and posteromedial cortex predicts memory performance in cognitively intact older individuals. Neurolmage, 51(2), 910-917.

Xia, Y., Chen, Q., Shi, L., Li, M., Gong, W., Chen, H., \& Qiu, J. (2019). Tracking the dynamic functional connectivity structure of the human brain across the adult lifespan. Human Brain Mapping, 40(3), 717-728.

Yeo, B. T. T., Krienen, F. M., Sepulcre, J., Sabuncu, M. R., Lashkari, D., Hollinshead, M., ... Buckner, R. L. (2011). The organization of the human cerebral cortex estimated by intrinsic functional connectivity. Journal of Neurophysiology, 106(3), 1125-1165.

Yin, D., Liu, W., Zeljic, K., Wang, Z., Lv, Q., Fan, M., ... Wang, Z. (2016). Dissociable Changes of Frontal and Parietal Cortices in Inherent Functional Flexibility across the 
Human Life Span. The Journal of Neuroscience: The Official Journal of the Society for Neuroscience, 36(39), 10060-10074.

Yu, M., Gouw, A. A., Hillebrand, A., Tijms, B. M., Stam, C. J., van Straaten, E. C. W., \& Pijnenburg, Y. A. L. (2016). Different functional connectivity and network topology in behavioral variant of frontotemporal dementia and Alzheimer's disease: an EEG study. Neurobiology of Aging, 42, 150-162.

Zhou, J., Greicius, M. D., Gennatas, E. D., Growdon, M. E., Jang, J. Y., Rabinovici, G. D., ... Seeley, W. W. (2010). Divergent network connectivity changes in behavioural variant frontotemporal dementia and Alzheimer's disease. Brain: A Journal of Neurology, 133(Pt 5), 1352-1367.

Zuo, X.-N., \& Xing, X.-X. (2014). Test-retest reliabilities of resting-state FMRI measurements in human brain functional connectomics: a systems neuroscience perspective. Neuroscience and Biobehavioral Reviews, 45, 100-118. 